\title{
FLT3 mutated acute myeloid leukemia: 2021 treatment algorithm
}

\author{
Naval Daver $\mathbb{D}^{1}$, Sangeetha Venugopal ${ }^{1}$ and Farhad Ravandi ${ }^{1}$
}

\begin{abstract}
Approximately $30 \%$ of patients with newly diagnosed acute myeloid leukemia (AML) harbor mutations in the fms-like tyrosine kinase 3 (FLT3) gene. While the adverse prognostic impact of FLT3-ITD ${ }^{\text {mut }}$ in AML has been clearly proven, the prognostic significance of FLT3-TKD ${ }^{\text {mut }}$ remains speculative. Current guidelines recommend rapid molecular testing for FLT3 ${ }^{\text {mut }}$ at diagnosis and earlier incorporation of targeted agents to achieve deeper remissions and early consideration for allogeneic stem cell transplant (ASCT). Mounting evidence suggests that FLT3 ${ }^{m u t}$ can emerge at any timepoint in the disease spectrum emphasizing the need for repetitive mutational testing not only at diagnosis but also at each relapse. The approval of multi-kinase FLT3 inhibitor (FLT3i) midostaurin with induction therapy for newly diagnosed FLT3 ${ }^{\text {mut }} A M L$, and a more specific, potent FLT3i, gilteritinib as monotherapy for relapsed/refractory (R/R) FLT3 ${ }^{\text {mut }} A M L$ have improved outcomes in patients with FLT3 ${ }^{\text {mut }}$ AML. Nevertheless, the short duration of remission with singleagent FLT3i's in R/R FLT3 ${ }^{\text {mut }}$ AML in the absence of ASCT, limited options in patients refractory to gilteritinib therapy, and diverse primary and secondary mechanisms of resistance to different FLT3i's remain ongoing challenges that compel the development and rapid implementation of multi-agent combinatorial or sequential therapies for FLT3 ${ }^{\text {mut }}$ AML.
\end{abstract}

\section{Overview}

Fms-like tyrosine kinase 3 (FLT3) is a recurrent genetic abnormality in AML $(\sim 30 \%)^{1-3}$. FLT3 activating mutations $\left(F L T 3^{\text {mut }}\right)$ may involve either the juxta membrane domain [internal tandem duplication mutations (FLT3ITD) $]^{4}$ or the tyrosine kinase domain (FLT3-TKD) $)^{5,6}$. In patients with newly diagnosed AML, FLT3-ITD ${ }^{\text {mut }}$ is a poor prognostic factor in terms of relapse-free (RFS) and overall survival $(\mathrm{OS})^{7-10}$. FLT3-TKD activating mutations also constitutively activate FLT ${ }^{11}$; however, they have not been associated with a consistent prognostic impact ${ }^{12}$.

\section{Prognostic impact of FLT3 mutations}

Not all FLT3-ITD ${ }^{\text {mut }}$ are equal; the prognostic impact is influenced by the allele ratio (AR), insertion site, ITD length, co-mutations (NPM1), and karyotype. $\mathrm{AR}$ is

\footnotetext{
Correspondence: Naval Daver (ndaver@mdanderson.org)

'Department of Leukemia, The University of Texas - MD Anderson Cancer Center, Houston, TX, USA

These authors contributed equally: Naval Daver, Sangeetha Venugopal
}

defined as the ratio of ITD-mutated alleles to wild-type allele (FLT3-ITD/FLT3 wild-type ${ }^{13}$. Variant allele frequency (VAF) is the ratio of ITD-mutated alleles to ITDmutated + wild-type alleles (FLT3-ITD/FLT3-ITD + FLT3 wild-type) ${ }^{14}$. Schlenk et al. evaluated the impact of AR in 323 patients with newly diagnosed FLT3-ITD ${ }^{\text {mut }}$ AML. After post-remission therapy with either consolidation (high-dose cytarabine-based) or allogeneic stem cell transplant (ASCT), AR $\geq 0.51$ and FLT3-ITD insertion site in TKD1 were associated with an unfavorable RFS $(P=0.0008)$ and $\mathrm{OS}(\mathrm{P}=0.004)^{15}$. In fact, every quartile increase in FLT3-ITD AR (from 0.01 to $0.20,0.20$ to 0.53 , 0.53 to $0.80,0.80$ to 1.19 ) was associated with worsening complete remission (CR) rates, RFS, and OS, highlighting the prognostic value of AR. It is important to note that none of these patients received a FLT3 inhibitor (FLT3i) during induction, consolidation, or post-ASCT.

The UKMRC group evaluated the presence of NPM1 co-mutations in young adult patients with AML. Favorable relapse risk and OS were seen in NPM1 ${ }^{\text {mut }}$ with FLT3 
wild-type; intermediate prognosis in FLT3-ITD ${ }^{\text {mut }}$ with concurrent $N P M 1^{\text {mut }}$, and adverse prognosis in FLT3ITD $^{\text {mut }}$ with NPM1 wild-type patients ${ }^{16}$. The Spanish group evaluated intermediate-risk AML patients treated with intensive chemotherapy. In patients with concurrent $N P M 1^{\text {mut }}$, the $\mathrm{OS}$ and relapse risk were comparable between FLT3 wild-type and FLT3-ITD ${ }^{\text {mut }} \mathrm{AR}<0.5$, but worse when $\mathrm{AR} \geq 0.5$. Among those with NPM1 wild-type, all FLT3-ITD ${ }^{\text {mut }}$ patients had an increased risk of relapse and inferior OS, regardless of the $\mathrm{AR}^{17}$. The current European Leukemia Net (ELN) guidelines categorize FLT3 -ITD ${ }^{\text {mut }}$ AML as favorable $\left(N P M 1^{\text {mut }}\right.$ with $F L T 3$ wild-type Or $N P M 1^{\text {mut }}$ with $F L T 3$-ITD $\left.\mathrm{AR}<0.5\right)$, intermediate $\left(N P M 1^{\text {mut }}\right.$ with $F L T 3-$ ITD AR $>0.5$ Or $N P M 1^{\text {WT }}$ with FLT3-ITD $\mathrm{AR}<0.5)$, or adverse $\left(N P M 1^{\mathrm{WT}}\right.$ with FLT3-ITD AR $>0.5)^{18}$. However, a subsequent UKMRC study of 1600 patients with cytogenetic intermediate-risk AML showed that relapse risk did not differ based on the FLT3-ITD ${ }^{\text {mut }} \mathrm{AR}$, and that the cumulative incidence of relapse in patients with $N P M 1^{\text {mut }}$ was increased with a concurrent FLT3-ITD ${ }^{\text {mut }}$ irrespective of the $\mathrm{AR}^{19}$. Oran et al. recently showed that ASCT in CR1 improved RFS and OS independent of the FLT3-ITD ${ }^{\text {mut }}$ AR or NPM1 ${ }^{\text {mut }}$ status in patients with $F L T 3-\mathrm{ITD}^{\text {mut }} \mathrm{AML}^{20}$. Collectively, $N P M 1^{\text {mut }}$ even with $F L T 3-\mathrm{ITD}^{\text {mut }}$ AR $<0.5$ are likely higher risk than truly "favorable risk" AML and we continue to consider them for ASCT in CR1.

Minetto and colleagues retrospectively evaluated the efficacy of fludarabine, high-dose cytarabine, and idarubicin (FAI) in 149 newly diagnosed FLT3-ITD ${ }^{\text {mut }}$ and/or $N P M 1^{\text {mut }}$ AML (only FLT3-ITD ${ }^{\text {mut }}=29 ; F L T 3-$ ITD $^{\text {mut }}$ $N P M 1^{\text {mut }}=59$, only $N P M 1^{\text {mut }}=61$ ). In patients $\leq 55$ years, this regimen appeared to overcome the negative impact of FLT3-ITD ${ }^{\text {mut }}$ in NPM1 co-mutated patients, regardless of the FLT3 AR, with comparable 3-year OS rates of $64 \%$ and $68 \%$ in $F L T 3-$ ITD $^{\text {mut }} N P M 1^{\text {mut }}$ and FLT3-ITD ${ }^{\mathrm{WT}} N P M 1^{\text {mut }}$ patients, respectively $(P>0.05)$. Moreover, ASCT in CR1 only benefitted patients with isolated FLT3-ITD ${ }^{\text {mut }}$ (without NPM1 ${ }^{\text {mut }}$ ) irrespective of AR $(P<0.05)^{21}$.

Taken together, utilizing baseline FLT3-ITD ${ }^{\text {mut }}$ AR to guide the post-remission therapy remains controversial. We currently recommend the incorporation of FLT3i's and ASCT in CR1 in all ASCT eligible patients with a FLT3-ITD ${ }^{\text {mut }}$ AML, irrespective of the AR and/or NPM1 co-mutation status. However, emerging data does suggest that patients with FLT3-ITD ${ }^{\text {mut }} \mathrm{AR}<0.5$ and NPM1 co-mutation without concurrent high-risk mutations such as DNMT3A, TP53, TET2, or high-risk cytogenetics may be a more favorable subset, who may be considered for induction, consolidation followed by maintenance therapy without ASCT on a case by case basis if they achieve early MRD negativity using a highly sensitive MRD assay.

\section{First-generation FLT3 inhibitors}

Type I FLT3i's are active against both the FLT3-ITD or TKD, type II inhibitors are only active against FLT3-ITD, not TKD. The first-generation FLT3i's lack specificity for FLT3 and inhibit multiple downstream RTKs that may result in more off-target toxicities. Second-generation FLT3i's potently and specifically target FLT3 with fewer off-target effects.

Midostaurin is a type I FLT3i active against PDGFR, KIT, SRC, and other RTKs ${ }^{22,23}$. In the randomized phase III RATIFY trial of midostaurin combined with cytarabine and daunorubicin $(3+7)$ induction and consolidation, midostaurin improved OS compared to placebo in patients $<60$ years of age with newly diagnosed FLT3 (ITD and/or TKD) $\mathrm{AML}^{24}$, regardless of $\mathrm{AR}(\leq 0.7$ or $\geq 0.7)$ or the type of mutation (ITD or TKD). Patients treated with midostaurin had higher rates of anemia and skin rash compared to placebo and these were generally manageable with supportive care without necessitating dose reductions or interruptions in the majority of cases. Pulmonary infiltration and acute pneumonitis-like picture are rare $(<1 \%)$ but noted side effects of midostaurin that treating physicians should be aware of. Midostaurin has been approved and widely used in combination with induction and consolidation therapy in patients with newly diagnosed $F L T 3^{\text {mut }} \mathrm{AML}^{25}$.

Sorafenib is a first generation, type II multi-kinase

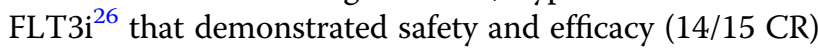
in combination with the standard anthracycline/cytarabine induction therapy in newly diagnosed $F L T 3^{\text {mut }}$ $\mathrm{AML}^{27}$. SORAML, a randomized placebo-controlled trial evaluated the efficacy and tolerability of $3+7$ inductionconsolidation with or without sorafenib in patients $\leq 60$ years with newly diagnosed AML, irrespective of a $F L T 3^{\text {mut }}$ (only $34 \%$ had $F L T 3^{\text {mut }}$ ). The addition of sorafenib significantly improved the event-free survival (EFS; 21 months vs 9 months; $P=0.013$ ) and RFS (56\% vs $38 \%$ ), but not $\mathrm{OS}^{28}$, although a recent update suggested an emerging trend toward improved $\mathrm{OS}^{29}$. The sorafenib treatment arm had increased rates of adverse events, particularly diarrhea, bleeding, cardiac events, hand-footskin reaction, and rash but with no significant increase in the 30- or 60-day mortality between the two treatment arms. Intriguingly, this was the first large study to show that the FLT3i may also benefit FLT3 wild-type patients, perhaps through multi-kinase blockade or prevention of emergent FLT3 clones at relapse ${ }^{28}$. Recently, a doubleblind placebo-controlled study reported a trend toward improved OS but not EFS with sorafenib combined with intensive chemotherapy in the frontline setting, especially among those with high FLT3-ITD ${ }^{\text {mut }}$ AR $>0.7^{30}$. Sorafenib with azacitidine combination reported an overall response rate (ORR) of $78 \%(n=27)$ in the frontline patients not eligible for intensive induction ${ }^{31}$ and an ORR of $46 \%$ with 
an acceptable safety profile in $\mathrm{R} / \mathrm{R}$ FLT3-ITD ${ }^{\text {mut } 32}$ which led to the inclusion of sorafenib with azacitidine combination as a $2 \mathrm{~B}$ guideline in National Comprehensive Cancer Network (NCCN) for R/R FLT3-ITD ${ }^{\text {mut }}$ AML $^{33}$.

\section{Second-generation FLT3 inhibitors}

Quizartinib, a second-generation, type I FLT3i is active against FLT3, KIT, CSF1R, PDGFR, and RET kinase ${ }^{34}$. Unlike midostaurin, quizartinib monotherapy, even at lower doses demonstrated significant marrow remissions in $\mathrm{R} / \mathrm{R} \mathrm{FLT3}{ }^{\mathrm{mut}} \mathrm{AML}^{35-37}$. In a single-arm phase II trial of quizartinib (90 or $135 \mathrm{mg}$ ), the CRc rates were between 46 and $56 \%$ in $\sim 250 \mathrm{R} / \mathrm{R}$ FLT3-ITD ${ }^{\text {mut }}$ patients treated across two cohorts. QTc prolongation $>500 \mathrm{~ms}$ emerged as a significant adverse event ${ }^{36}$. A subsequent randomized phase IIb trial evaluated lower doses, 30 or $60 \mathrm{mg}$ of quizartinib daily, in patients with R/R FLT3-ITD ${ }^{\text {mut }}$ AML. The CRc rates (47\%) were similar between both doses, and the frequency of QTcF $>500 \mathrm{~ms}$ was significantly reduced (3-5\%) with these lower doses of quizartinib ${ }^{35}$.

QuANTUM-R, a phase 3 randomized controlled trial, evaluated quizartinib monotherapy vs investigator choice salvage chemotherapy in R/R FLT3-ITD ${ }^{\text {mut }}$ AML. Quizartinib demonstrated an OS of 6.2 months compared with 4.7 months with salvage chemotherapy (hazard ratio 0.76 and $P=0.02$ ). The $C R c$ rates with quizartinib were similar to prior studies (48.2\%), and $32 \%$ patients on the quizartinib arm underwent ASCT compared with $11 \%$ with salvage chemotherapy. Given the magnitude of OS benefit and concerns over therapeutic equipoise and potential cardiac safety signals, quizartinib was not approved in the US and Europe, but approved in Japan as a monotherapy in $\mathrm{R} / \mathrm{R}$ FLT3-ITD ${ }^{\text {mut }}$ AML. In general, quizartinib is well tolerated with minimal skin, gastrointestinal, or pulmonary side effects. However, in addition to QTcF prolongation, quizartinib is also more myelosuppressive than many other FLT3 inhibitors likely due to the inhibition of KIT.

Gilteritinib, a second-generation type I FLT3i demonstrated tolerability with CRc rates of $45-55 \%$ in patients with $\mathrm{R} / \mathrm{R}$ FLT3 (ITD or TKD) ${ }^{\mathrm{mut}} \mathrm{AML}^{38,39}$. The randomized phase III ADMIRAL trial evaluated gilteritinib vs investigator choice salvage chemotherapy in patients with $\mathrm{R} / \mathrm{R} F L T 3^{\mathrm{mut}}$ AML. Gilteritinib decreased the risk of death by $36 \%$ compared with salvage chemotherapy, with a median OS of 9.3 months vs 5.6 months $(P<0.001)$, and a superior $\mathrm{CR}+\mathrm{CRh}$ rate (34\% vs $15.3 \%)$. Gilteritinib was generally well tolerated but was associated with increased incidence of gastrointestinal side effects, most frequently diarrhea although nausea has been occasionally observed. Increase in bilirubin and transaminase can be seen with giltertiinib but are usually self-resolving and transient. Posterior reversible encephalopathy and pancreatitis are rare $(<1-2 \%)$ but important side effects to be aware of.
These results led to the approval of gilteritinib monotherapy in the US and Europe in patients with $R / R$ FLT3 $^{\text {mut }} \mathrm{AML}^{40}$.

\section{Post-ASCT maintenance with FLT3 inhibitors}

In patients with $F L T 3^{\text {mut }} A M L$ who relapsed after first ASCT, sorafenib was found to be tolerable with longlasting remissions in 7 of 29 patients treated, suggesting a potential synergy with post-ASCT alloimmune effects ${ }^{41}$. SORMAIN, a placebo-controlled randomized phase II trial evaluated post-transplant sorafenib maintenance in patients with FLT3-ITD ${ }^{\text {mut }}$ AML with RFS post-ASCT as the primary endpoint. At a median follow-up of 42 months, sorafenib demonstrated a 2-year estimated RFS of $85 \%$ and OS of $90.5 \%$ compared with $53.3 \%(P=$ $0.002)$, and $66.2 \%$ with placebo, respectively $(P=0.007)$. Although the toxicity-related discontinuation rate was low (22\%), sorafenib-treated patients did experience higher rates of graft-versus-host disease (GVHD) and skin toxicity $^{42}$. In another randomized phase III study comparing post-ASCT sorafenib maintenance $(n=100)$ to non-maintenance $(n=102)$, sorafenib demonstrated an improved 1 -year OS $(82.1 \%$ vs $68 \%, P=0.012)$ and a decreased 1-year cumulative incidence of relapse $(7 \%$ vs $24.5 \%, P=0.001)$ in $F L T 3-I T D^{\text {mut }}$ AML patients undergoing ASCT in CR1 ${ }^{43}$. We currently recommend posttransplant maintenance with a FLT3i for at least 2 years (potentially indefinitely as there is limited data on the incidence of possible late relapses) in all $F L T 3^{\text {mut }}$ AML. The MORPHO phase III placebo-controlled trial evaluating post-transplant maintenance with gilteritinib in $F L T 3^{\text {mut }}$ AML recently completed accrual and results are eagerly awaited (NCT02997202).

\section{Moving forward to maximize benefit: FLT3 inhibitors combination therapy}

Frontline FLT3i's with anthracycline/cytarabine induction or hypomethylating agents (HMAs)

A phase I study evaluating gilteritinib with $7+3$ induction and high-dose cytarabine consolidation chemotherapy, followed by single-agent maintenance therapy, in patients with newly diagnosed AML showed that gilteritinib $120 \mathrm{mg}$ daily was well tolerated. Among 38 patients with $F L T 3^{\text {mut }}$ AML who received gilteritinib $120 \mathrm{mg}$ daily, the CRc rate was $81.6 \%(n=31)$ including $39.5 \%$ CR and median OS was not reached at a median follow-up of 35.8 months. Two randomized trials are evaluating the addition of gilteritinib vs midostaurin to induction and consolidation therapy in patients with newly diagnosed $F L T 3^{\text {mut }}$ AML $^{44}$ (NCT04027309, NCT03836209).

The LACEWING phase III randomized trial evaluated gilteritinib with azacitidine vs azacitidine monotherapy (NCT02752035) in patients with newly diagnosed 
$F L T 3^{\text {mut }}$ AML not eligible for intensive induction chemotherapy. The CRc rate was $67 \%(n=10 / 15)$ in the combination arm in the safety cohort prior to commencement of randomization ${ }^{45}$. However, in a recently released planned interim analysis, the study did not meet its primary endpoint of overall survival and may be terminated for futility ${ }^{46}$.

Strati et al. evaluated midostaurin with azacitidine in patients with both newly diagnosed and R/R AML regardless of FLT3 mutational status. Among the $F L T 3^{\text {mut }}$ patients, response rates were numerically higher (33\%) and remission duration was longer (31 versus 16 weeks, $P$ $=0.09$ ) in those who were naive to treatment with FLT3 inhibitors compared with those who had been exposed to prior FLT3 inhibitors. Although activity was seen, the response rates were overall modest with this combination and the combination of HMA with midostaurin is not one that we routinely use or recommend for frontline FLT3mutated $\mathrm{AML}^{47}$.

Swaminathan et al. evaluated quizartinib (60 mg daily) combined with either azacitidine or low-dose cytarabine in patients with newly diagnosed or R/R FLT3 ${ }^{\text {mut }}$ AML not eligible for intensive chemotherapy. Among patients treated with azacitidine and quizartinib in the frontline setting, the CRc rate was $78 \%(n=7 / 9)$ with a median OS of 21.1 months. In the R/R setting, the CRc rate was $64 \%$ ( $n=18 / 28)$ with a median OS of 12.0 months, with responses observed even in prior FLT3i exposed patients $^{48}$. A randomized, placebo-controlled phase III study of $3+7$ with quizartinib (QuANTUM-First; NCT02668653) in patients with newly diagnosed FLT3$I T D^{\text {mut }}$ AML eligible for induction therapy recently completed accrual. Quizartinib is also being evaluated in combination with CPX-351 (NCT04209725) and with CLIA (NCT04047641) in treatment naive and $R / R$ $F L T 3^{\text {mut }}$ AML.

\section{Combinations with venetoclax with or without HMA}

Based on the strong preclinical synergy and synthetic lethality with venetoclax and FLT3i combination ${ }^{49-51}$, and the fact that BCL2 upregulation may confer resistance to FLT3 inhibition ${ }^{52}$, evaluation of several doublet and triplet combinations of venetoclax and FLT3i are ongoing. Gilteritinib with venetoclax (NCT03625505) was evaluated in 41 patients with heavily pretreated R/R FLT3 ${ }^{\text {mut }}$ AML (median salvage 2, 65\% previously exposed to FLT3i) ${ }^{40,53}$. Using the same response criteria, the CRc rate was $85.4 \%(n=35 / 41)$ which compared favorably to $52 \%$ with gilteritinib alone in the ADMIRAL study. However, the true CR/CRi rate was only $34 \%$. Molecular clearance of FLT3 was noted in $50 \%$ of all evaluable patients. Encouragingly, the response rate was maintained among patients previously exposed to other FLT3 TKIs. The combination continues to enroll.
Maiti et al. recently presented the first triplet combination of venetoclax, FLT3i (mainly gilteritinib or sorafenib), and decitabine from the $F L T 3^{\text {mut }}$ subset of the prospective decitabine 10 days with venetoclax study $(\mathrm{NCT} 03404193)^{54}$. Among 16 patients with newly diagnosed $F L T 3^{\text {mut }}$ AML not eligible for intensive induction, the CRc rate was $88 \%$ with FLT3-PCR negativity in $100 \%$ of responders and a projected 2-year OS of $>80 \%$. Among $14 \mathrm{R} / \mathrm{R} F L T 3^{\mathrm{mut}} \mathrm{AML}$ patients, the CRc rate was $64 \%$ with FLT3-PCR negativity in $88 \%$ of responders. In the treatment-naive setting, the median time to neutrophil and platelet recovery among responders was 45 and 30 days, respectively, suggesting cumulative myelosuppression is to be expected and further optimization of triplets schedules is ongoing ${ }^{55}$.

Yilmaz et al. prospectively evaluated decitabine and quizartinib (doublet) with or without venetoclax (triplet) in patients with newly diagnosed and R/R FLT3-ITD ${ }^{\text {mut }}$ AML. While the seven patients treated with the doublet had a CRc rate of $57 \%(n=4 / 7)$ and a median OS of 5.7 months, the fifteen R/R $F L T 3^{\text {mut }}$ AML patients treated with the triplet had a CRc rate of $81 \%(n=11)$ with a projected 1-year OS of $60 \%$. In the frontline setting $(n=$ 4), the CRc rate with the triplet was $100 \%$ with FLT3-PCR negativity in all four patients ${ }^{56}$.

These data highlight the potent anti-leukemic activity of the triplet approach in $F L T 3^{\text {mut }}$ AML. We believe that triplets may be the optimal way to use FLT3i to improve long-term survival and "cure rates" in older patients, able to tolerate this approach. Further evaluation and optimization of triplets is a major area of clinical research focus in $F L T 3^{\text {mut }}$ AML.

\section{Treatment algorithm of FLT3-mutated AML}

Clinical trial enrollment (if available) is always the first option, in both frontline and R/R FLT3 ${ }^{\text {mut }}$ AML. The choice of treatment backbone depends on the patient's ability to successfully tolerate intensive chemotherapy. Accumulating evidence have shown improved outcomes in FLT3-ITD ${ }^{\text {mut }}$ patients receiving induction with higher dose anthracyclines ${ }^{57}$, cladribine ${ }^{58}$, or fludarabine added to induction backbone ${ }^{21}$, and incorporating FLT3i with induction (either first or second generation) in $F L T 3^{\text {mut }}$ $\mathrm{AML}^{24,44,59,60}$ (Fig. 1A). Our treatment approach for $F L T 3^{\text {mut }}$ AML in MD Anderson Cancer Center is as follows: in newly diagnosed patients who are eligible to receive intensive chemotherapy (Fig. 1B) we add a second generation FLT3i to the intensive induction backbone of cladribine or fludarabine with cytarabine and idarubicin (CLIA or FIA, respectively) as published previously by our group $^{61,62}$. Addition of venetoclax to this backbone may be associated with prolonged and potentially prohibitive myelosuppression; we have not routinely added and do not at this time recommend adding venetoclax to the 

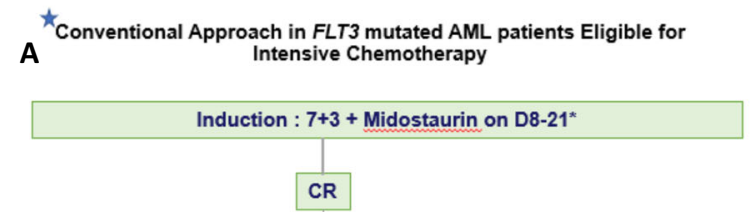

Age, ECOG PS, comorbidities, baseline CG and molecular, MRD status, donor

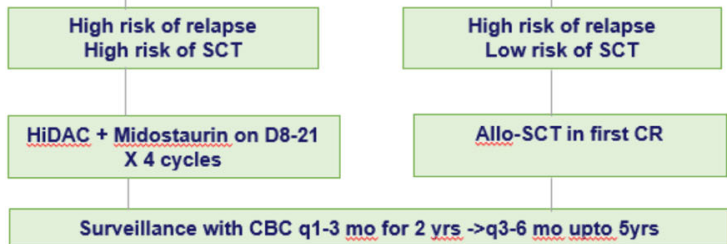

Surveillance with CBC q1-3 mo for 2 yrs $\rightarrow$ q3-6 mo upto $5 y r s$

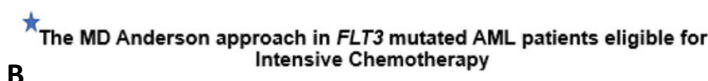

B

Induction: FAI/CLIA + second generation FLT3i D1-14 consolidation $\times 1-2$ cycles

CR

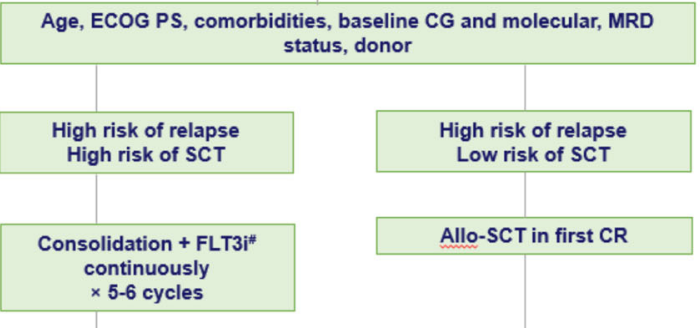

Maintenance FLT3i (+/-HMA) × 2 yrs @

$\downarrow$ Assess patients for clinical trial options

*- Bone marrow assessment on D21

*We prefer second generation FLT3 inhibitor for our patients in all settings.

We advocate maintenance therapy with HMA and FLT3i combination in young and fit patients not eligible for Allogenic Stem Cell Transplant (ASCT)

@|deal duration of FLT3i maintenance is not defined but we recommend at least 2 years maintenance, and in most cases prefer indefinite maintenance if good tolerability

Fig. 1 Treatment algorithm of FLT3-ITD-mutated AML in patients eligible for intensive chemotherapy. A Conventional approach. B MD Anderson Cancer Center Approach. 7+3-7 days of cytarabine and 3 days of daunorubicin. F fludarabine, I idarubicin, CL cladribine, A cytarabine $1.5-2 \mathrm{~g} / \mathrm{m}^{2}$, HMA hypomethylating agent, CR complete remission, ECOG PS Eastern Cooperative Oncology Group Performance Status, CG cytogenetics, MRD measurable residual disease, SCT stem cell transplant, HiDAC high-dose cytarabine, CBC complete blood count.

backbone of CLIA/FIA with FLT3i ${ }^{63}$. We prefer a secondgeneration FLT3i (ideally gilteritinib) in the newly diagnosed setting, and administer the FLT3i D1-D14 during induction, and continuously starting Cycle 2 Day 1 through consolidation.

Upon achieving CR, the decision for ASCT is based on the risk-benefit assessment for ASCT. All eligible intermediate or high-risk patients (defined as patients with FLT3-ITD ${ }^{\text {mut }}$ AR $>0.50$ irrespective of NPM1 ${ }^{\text {mut }}$ status, or FLT3-ITD ${ }^{\text {mut }} \mathrm{AR}<0.50$ without $N P M 1^{\text {mut }}$ ) are equivocally recommended to proceed to ASCT in CR1 followed by post-ASCT FLT3i maintenance for at least 2 years (although we often continue indefinite FLT3i maintenance until long-term maintenance data becomes available). For post-ASCT maintenance, our agent of choice has been gilteritinib $80-120 \mathrm{mg}$ day either as a single agent or combined with low-dose azacitidine. The role of ASCT in patients with $F L T 3-\mathrm{ITD}^{\text {mut }} \mathrm{AR}<0.50$ with concomitant $N P M 1^{\text {mut }}$ in the absence of concomitant high-risk features such as DNMT3A, TP53, or RUNX1 co-mutations, adverse cytogenetics, therapy-related or secondary AML, who achieve MRD negativity by high-sensitivity PCR (ideally for $N P M 1^{\mathrm{mut}}$ ), or patients with $F L T 3-\mathrm{TKD}^{\mathrm{mut}}$ is an area of ongoing debate. We evaluate these patients on a case by case basis and may consider maintenance with 4-5 consolidation cycles of CLIA or FAI with FLT3i followed by FLT3i +/- HMA maintenance for two years vs ASCT based on donor availability, age, performance status, MRD negativity, and patient preference.
In patients with $F L T 3^{\text {mut }}$ AML unsuitable for intensive chemotherapy, azacitidine with venetoclax demonstrated encouraging $\mathrm{CR} / \mathrm{CRi}$ rates $(55-70 \%)$ and a median OS of 13.3 months $^{64}$ which prompted the inclusion of this combination approach as part of NCCN AML guidelines (Fig. 2A). However, the median OS was 19.2 months in FLT3-TKD ${ }^{\text {mut }}$ AML (19.2 months), but only 11.5 months in FLT3-ITD ${ }^{\text {mut }}$ patients ${ }^{65}$. This is in line with the preclinical data ${ }^{49}$ and molecular profiling of pre- and posttreatment samples ${ }^{66}$ identifying $F L T 3-$ ITD $^{\text {mut }}$ as a putative mechanism of resistance to venetoclax based therapies $^{67}$, suggesting that FLT3-ITD ${ }^{\text {mut }}$ patients may need a FLT3i incorporated into the HMA with venetoclax therapy either in a triplet or sequential approach to improve OS. Therefore, in patients not eligible for intensive chemotherapy at MDACC, we prefer a combination of HMA with venetoclax and FLT3i (gilteritinib) over an HMA with venetoclax doublet (Fig. 2B). Administration of the triplet is associated with prolonged cytopenias, requiring close monitoring and experience with venetoclax based combinations.

We administer a second-generation FLT3i (ideally gilteritinib) continuously with HMA from cycle 1 Day 1 . We introduce venetoclax with a ramp-up when the WBC is $<10,000 / \mu \mathrm{L}$ to decrease the risk of tumor lysis syndrome. To mitigate prolonged myelosuppression with the triplet and avoid over-treatment, we perform an early bone marrow assessment on Cycle 1 Day 14 (Fig. 3). We stop the venetoclax and the FLT3i after Day 14 in patients who 


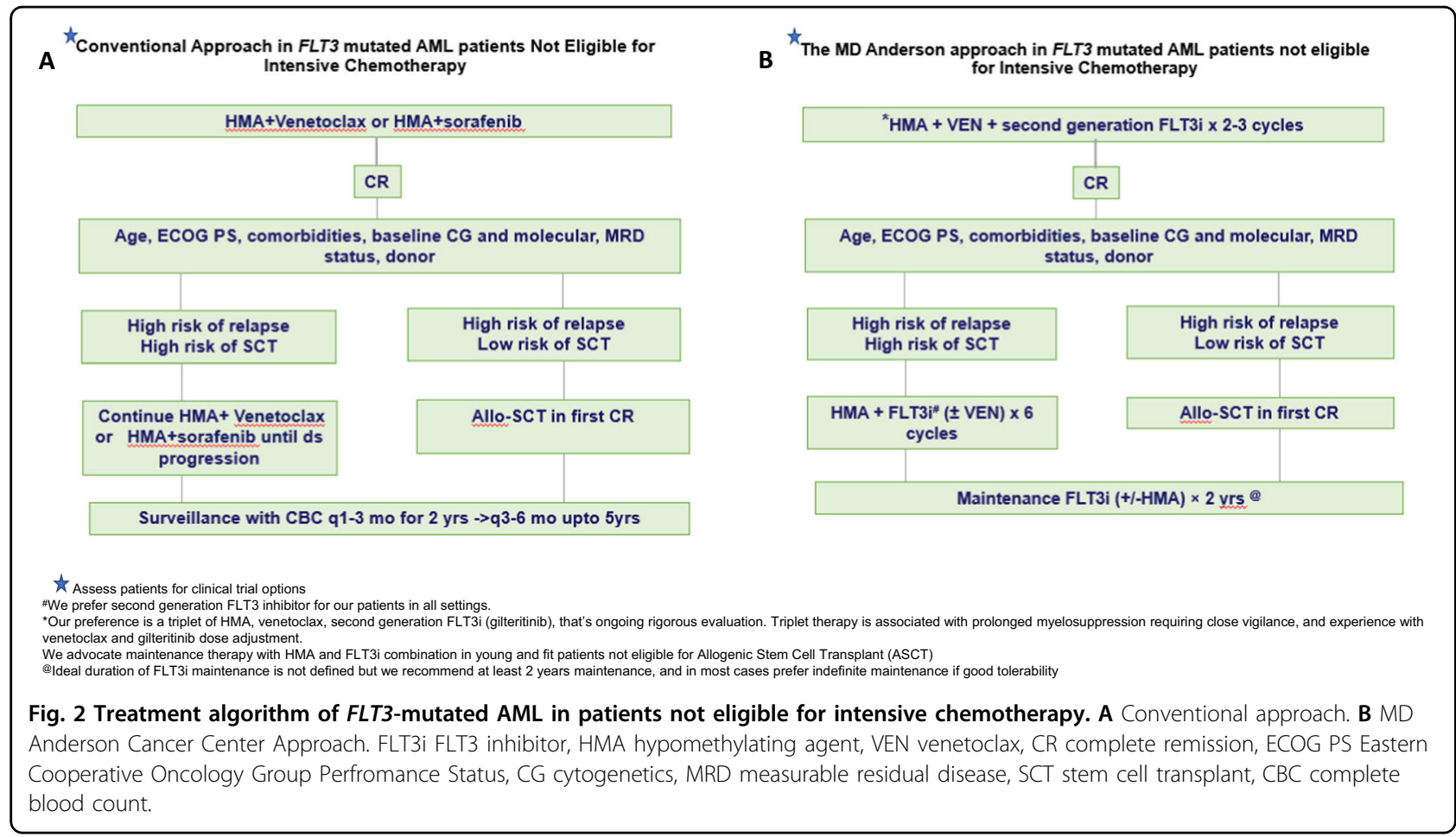

\section{Initial response assessment with the triplet regimen}

Cycle 1

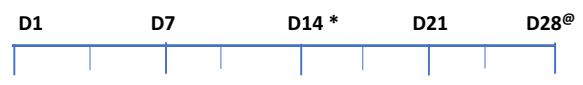

D1-5

DAC $20 \mathrm{mg} / \mathrm{m}^{2}$

OR

AZA $75 \mathrm{mg} / \mathrm{m}^{2}$ $\stackrel{+}{+} \underset{\text { Start }}{2^{\text {nd }}}$ gen

FLT3i

Start Venetoclax

when $\mathrm{WBC}<10 \mathrm{~K}$

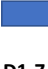

D1-7

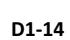

D1-14

D1-14
* up to D21

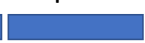

** up to D21

- *C1 D14: Perform bone marrow biopsy; If bone marrow shows $<5 \%$ blasts and/or $<5 \%$ cel

- **If the C1 D14 bone marrow show $>5 \%$ blasts $\rightarrow>$ continue venetoclax and FLT3i till D21

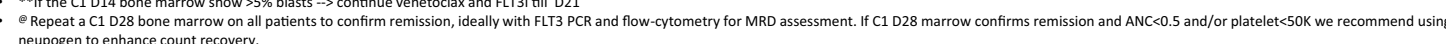

Fig. 3 Initial response assessment with the triplet regimen. ${ }^{*} \mathrm{C} 1$ D14: Perform bone marrow biopsy; if bone marrow shows $<5 \%$ blasts and/or $<5 \%$ cellularity/insufficient sample $\rightarrow$ Stop venetoclax and FLT3i on D14. ${ }^{*}$ If the C1 D14 bone marrow show $>5 \%$ blasts $\rightarrow$ continue venetoclax, FLT3i till D21. ${ }^{\circledR}$ Repeat a C1 D28 bone marrow on all patients to confirm remission. If C1 D28 marrow confirms remission and ANC $<0.5$ and/or platelet $<$ 50K consider interrupting FLT3i and using neupogen to enhance count recovery.

achieve marrow remission $(<5 \%$ blasts $)$ and/or marrow aplasia/hypoplasia/insufficiency $(<5 \%$ cellularity $)$. We continue the venetoclax and FLT3i until Day 21 if the Day 14 bone marrow shows $>5 \%$ blasts with $>/=5 \%$ cellularity. In patients with ongoing cytopenias $(\mathrm{ANC}</=0.5$ and/or platelets $</=50 \mathrm{~K})$ on Day 28, we repeat a bone marrow on
Day 28 to confirm marrow remission and once confirmed recommend administering growth factors starting Day 28 to boost recovery. In subsequent cycles: FLT3i is continued for the entire duration of the cycle and the venetoclax duration is reduced to 14 days or lower to mitigate cumulative prolonged cytopenias. Although the triplet 


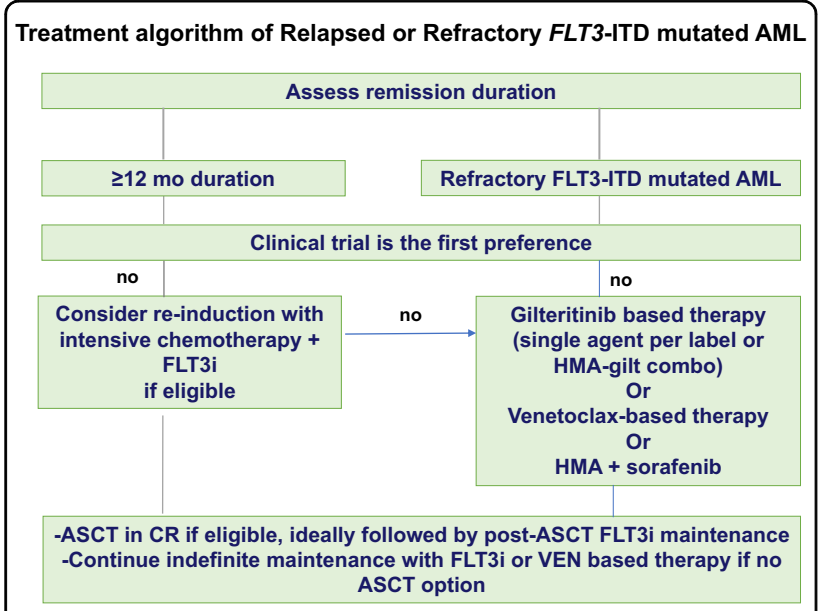

Fig. 4 Treatment algorithm of relapsed or refractory $F L T 3^{\text {mut }}$ AML. SCT stem cell transplant.

approaches are still in development, emerging data with the triplets as discussed previously, suggest rapid and high potency, deep molecular remissions, and encouraging survival. An alternate option would be to consider sequencing with alternate cycles of HMA with venetoclax and HMA with FLT3i. Such sequential approaches need to be formally evaluated in the context of prospective clinical trials.

In patients with relapsed or refractory $F L T 3^{\text {mut }} \mathrm{AML}$ (Fig. 4), diligent effort must be made to refer the patient to large academic centers with clinical trial options as the outcomes remain dismal with a median OS $<10$ months with almost any approach. In the absence of clinical trial options: among patients eligible for intensive chemotherapy who had a prior remission $>10-12$ months, we would prefer a regimen incorporating intensive therapy (FLAGIda, CLAG-M, CLIA, MEC) in combination with a FLT3 inhibitor with an intent to achieve a rapid and hopefully deep remission and transition patients to ASCT followed by post-ASCT maintenance. In older patients not eligible for intensive therapy, patients with primary refractory disease or early relapse with a persistent FLT3 mutation we would suggest gilteritinib based therapy. Although the label indication for gilteritinib is as a single agent we have never used it as a single agent but always in combination with either HMA alone, venetoclax alone or as a triplet with HMA and venetoclax. These combinations appear to improve the efficacy over single agent gilteritinib and could be considered if there is expertise in using such an approach, For patients relapsing while on gilteritinib or soon after gilteritinib based therapy a combination of azacitidine with sorafenib or azacitinde with venetoclax or gemtuzumab based approaches may be considered as salvage options (with clinical trials being clearly the best option if available).

\section{Mechanisms of resistance to FLT3 inhibitors}

Despite the encouraging development of FLT3i, resistance to FLT3i is not uncommon and it can be either primary or secondary. The primary resistance mechanisms include specific FLT3-TKD mutations (either single TKD mutations or compound mutations within the FLT3-ITD allele), mutations in genes other than FLT3, activation of alternative signaling pathways in leukemic cells or the bone marrow microenvironment that confer resistance to FLT3i ${ }^{68}$.

Secondary resistance to FLT3i could be either on-target (changes in the FLT3) or off-target (constitutive activation of non-FLT3-dependent oncogenic pathways). The ontarget mechanism of resistance includes emergence of secondary TKD mutations in patients treated with type II inhibitors like quizartinib or sorafenib ${ }^{69,70}$. Type I FLT3i's like gilteritinib are less prone to develop secondary mutations in the TKD, although the gatekeeper F691M can confer resistance to gilteritinib ${ }^{71}$. Off-target resistance includes clonal evolution during FLT3i therapy even when FLT3-ITD ${ }^{\text {mut }}$ clone is lost $^{70}$. In a study that identified molecular mechanisms of resistance to gilteritinib, $32 \%$ of patients had emergent mutations in the RAS/MAPK pathway (K/NRAS), and $5 \%$ had emergent $B C R / A B L 1$ fusions $^{71}$. More recently, the emergence of $B C R-A B L 1$ positive clone was shown as a resistance mechanism to multiple FLT3i's ${ }^{72}$. It is important to acknowledge the diverse mechanisms of FLT3i resistance after different FLT3i's, and it is essential to proactively evaluate for these mechanisms at the time of FLT3i failure to optimize subsequent therapy.

\section{Future direction}

In the QuANTUM-R and ADMIRAL trials, only $4 \%$ and $12 \%$ of patients had received prior FLT3i therapy with induction, making it difficult to draw conclusions regarding the outcomes of contemporary patients, most of whom will have received a FLT3i (commonly midostaurin) with induction ${ }^{36,40}$. Yilmaz et al. evaluated the outcomes of sequential FLT3i-based therapies in $F L T 3^{\text {mut }}$ AML. In the frontline setting, there was a sequential decrease in CRc rates $(77 \% \rightarrow 31 \% \rightarrow 25 \%)$ and OS $(16.7 \rightarrow 6.0 \rightarrow 1.4$ months). A comparable decrease in CRc rates $(45 \% \rightarrow 21 \% \rightarrow 10 \%)$ and OS $(7.9 \rightarrow 4.0 \rightarrow 4.1$ months $)$ was observed with sequential FLT3i-based therapies in the R/R AML setting ${ }^{73}$. Perl and colleagues investigated whether prior FLT3i therapy influenced outcomes in patients treated with gilteritinib. Regardless of prior FLT3i therapy, gilteritinib-treated patients had CRc rates $>40 \%$, however, the median OS with single-agent gilteritinib was 6.5 vs 9.6 months in prior FLT3i exposed $(n=31)$ vs naive patients $(n=216)$ with $F L T 3^{\text {mut }} \mathrm{R} / \mathrm{R} \mathrm{AML}^{74}$. These data suggests that although responses may still be achieved with gilteritinib in patients refractory to prior first- 
generation FLT3i-based therapies, optimization with doublet or triplet combinations with second-generation FLT3i is likely needed to significantly improve OS with prior TKI exposure.

\section{Funding}

This work was supported in part by the MD Anderson Cancer Centre Support Grant (CCSG) CA016672, the MD Anderson Cancer Center Leukemia SPORE CA100632, the Charif. Souki Cancer Research Fund and generous philanthropic contributions to the MD Anderson Moon Shots Program.

\section{Conflict of interest}

N.D. has received research funding from Daiichi-Sankyo, Bristol-Myers Squibb, Pfizer, Gilead, Sevier, Genentech, Astellas, Daiichi-Sankyo, Abbvie, Hanmi, Trovagene, FATE, Amgen, Novimmune, Glycomimetics, and ImmunoGen and has served in a consulting or advisory role for Daiichi-Sankyo, Bristol-Myers Squibb, Pfizer, Novartis, Celgene, AbbVie, Astellas, Genentech, Immunogen, Servier, Syndax, Trillium, Gilead, Amgen, and Agios. F.R. has received research funding from Astellas, and Novartis and has served as a member of advisory board in Astellas and Novartis. S.V. has nothing to disclose.

\section{Publisher's note}

Springer Nature remains neutral with regard to jurisdictional claims in published maps and institutional affliations.

Received: 31 December 2020 Revised: 22 April 2021 Accepted: 5 May 2021 Published online: 27 May 2021

\section{References}

1. Rosnet, O. et al. Expression and signal transduction of the FLT3 tyrosine kinase receptor. Acta Haematol. 95, 218-223 (1996).

2. Timothy, J. \& Ley, C., Network CGAR. Genomic and epigenomic landscapes of adult de novo acute myeloid leukemia. N. Engl. J. Med. 368, 2059-2074 (2013).

3. Welch John, S. et al. The origin and evolution of mutations in acute myeloid leukemia. Cell 150, 264-278 (2012).

4. Nakao, M. et al. Internal tandem duplication of the flt3 gene found in acute myeloid leukemia. Leukemia 10, 1911-1918 (1996).

5. Yamamoto, Y. et al. Activating mutation of $\mathrm{D} 835$ within the activation loop of FLT3 in human hematologic malignancies. Blood 97, 2434-2439 (2001).

6. Abu-Duhier, F. et al. Identification of novel FLT-3 Asp835 mutations in adult acute myeloid leukaemia. Br. J. Haematol. 113, 983-988 (2001).

7. Kiyoi, H. et al. Prognostic implication of FLT3 and N-RAS gene mutations in acute myeloid leukemia. Blood 93, 3074-3080 (1999).

8. Kottaridis, P. D. et al. Studies of FLT3 mutations in paired presentation and relapse samples from patients with acute myeloid leukemia: implications for the role of FLT3 mutations in leukemogenesis, minimal residual disease detection, and possible therapy with FLT3 inhibitors. Blood 100, 2393-2398 (2002).

9. Thiede, C. et al. Analysis of FLT3-activating mutations in 979 patients with acute myelogenous leukemia: association with $F A B$ subtypes and identification of subgroups with poor prognosis. Presented in part at the 42nd Annual Meeting of the American Society of Hematology, December 1-5, 2000, San Francisco, CA (abstract 2334). Blood 99, 4326-4335 (2002).

10. Fröhling, S. et al. Prognostic significance of activating FLT3 mutations in younger adults (16 to 60 years) with acute myeloid leukemia and normal cytogenetics: a study of the AML Study Group UIm. Blood 100, 4372-4380 (2002).

11. Kiyoi, H., Ohno, R., Ueda, R., Saito, H. \& Naoe, T. Mechanism of constitutive activation of FLT3 with internal tandem duplication in the juxtamembrane domain. Oncogene 21, 2555-2563 (2002).

12. Mead, A. J. et al. FLT3 tyrosine kinase domain mutations are biologically distinct from and have a significantly more favorable prognosis than FLT3 internal tandem duplications in patients with acute myeloid leukemia. Blood 110, $1262-1270$ (2007).
13. Whitman, S. P. et al. Absence of the wild-type allele predicts poor prognosis in adult de novo acute myeloid leukemia with normal cytogenetics and the internal tandem duplication of FLT3: a cancer and leukemia group B study. Cancer Res. 61, 7233-7239 (2001).

14. Yalniz, F. et al. Prognostic significance of baseline FLT3-TTD mutant allele level in acute myeloid leukemia treated with intensive chemotherapy with/without sorafenib. Am. J. Hematol. 94, 984-991 (2019).

15. Schlenk, R. F. et al. Differential impact of allelic ratio and insertion site in FLT3ITD-positive AML with respect to allogeneic transplantation. Blood 124, 3441-3449 (2014).

16. Gale, R. E. et al. The impact of FLT3 internal tandem duplication mutant level, number, size, and interaction with NPM1 mutations in a large cohort of young adult patients with acute myeloid leukemia. Blood 111, 2776-2784 (2008).

17. Pratcorona, M. et al. Favorable outcome of patients with acute myeloid leukemia harboring a low-allelic burden FLT3-ITD mutation and concomitant NPM1 mutation: relevance to post-remission therapy. Blood 121, 2734-2738 (2013).

18. Döhner, H. et al. Diagnosis and management of AML in adults: 2017 ELN recommendations from an international expert panel. Blood 129, 424-447 (2017).

19. Linch, D. C., Hills, R. K., Burnett, A. K, Khwaja, A. \& Gale, R. E. Impact of FLT3ITD mutant allele level on relapse risk in intermediate-risk acute myeloid leukemia. Blood 124, 273-276 (2014).

20. Oran, B. et al. Allogeneic transplantation in first remission improves outcomes irrespective of FLT3-ITD allelic ratio in FLT3-ITD-positive acute myelogenous leukemia. Biol. Blood Marrow Transplant 22, 1218-1226 (2016).

21. Minetto, P. et al. Intensive fludarabine, high dose cytarabine and idarubicinbased induction for younger NPM1-mutated AML patient: overcoming the negative prognosis of FLT3-ITD mutation. Blood 136, 32-33 (2020).

22. Tamaoki, T. et al. Staurosporine, a potent inhibitor of phospholipid $\mathrm{Ca}^{++}$ dependent protein kinase. Biochem. Biophys. Res. Commun. 135, 397-402 (1986).

23. Weisberg, E. et al. Inhibition of mutant FLT3 receptors in leukemia cells by the small molecule tyrosine kinase inhibitor PKC412. Cancer Cell 1, 433-443 (2002).

24. Stone, R. M. et al. Midostaurin plus chemotherapy for acute myeloid leukemia with a FLT3 mutation. N. Engl. J. Med. 377, 454-464 (2017).

25. Rydapt Prescribing Information. https:/www.accessdata.fda.gov/drugsatfda _docs/label/2017/207997s000lbl.pdf2017 (2017).

26. Žhang, W. et al. Mutant FLT3: a direct target of sorafenib in acute myelogenous leukemia. J. Natl Cancer Inst. 100, 184-198 (2008).

27. Ravandi, F. et al. Phase $1 / \|$ study of combination therapy with sorafenib, idarubicin, and cytarabine in younger patients with acute myeloid leukemia. J. Clin. Oncol. 28, 1856 (2010).

28. Röllig, C. et al. Addition of sorafenib versus placebo to standard therapy in patients aged 60 years or younger with newly diagnosed acute myeloid leukaemia (SORAML): a multicentre, phase 2, randomised controlled trial. Lancet Oncol. 16, 1691-1699 (2015).

29. Rollig, C. et al. The addition of sorafenib to standard AML treatment results in a substantial reduction in relapse risk and improved survival. Updated results from long-term follow-up of the randomized-controlled SORAML trial. Blood 130, 721 (2017)

30. Andrew, H. et al. Results of a Phase 2, Randomized, Double-Blind Study of Sorafenib Versus Placebo in Combination with Intensive Chemotherapy in Previously Untreated Patients with FLT3-ITD Acute Myeloid Leukemia (ALLG AMLM16) (ASH, 2020).

31. Ohanian, M. et al. Sorafenib combined with 5-azacytidine in older patients with untreated FLT3-ITD mutated acute myeloid leukemia. Am. J. Hematol. 93, 1136-1141 (2018)

32. Ravandi, F. et al. Phase 2 study of azacytidine plus sorafenib in patients with acute myeloid leukemia and FLT-3 internal tandem duplication mutation. Blood 121, 4655-4662 (2013).

33. Tallman, M. S. et al. Acute myeloid leukemia, Version 3.2019, NCCN clinical practice guidelines in oncology. J. Natl Compr. Canc. Netw. 17, 721-749 (2019).

34. Zarrinkar, P. P. et al. AC220 is a uniquely potent and selective inhibitor of FLT3 for the treatment of acute myeloid leukemia (AML). Blood 114, 2984-2992 (2009).

35. Cortes, J. E. et al. Phase $2 \mathrm{~b}$ study of 2 dosing regimens of quizartinib monotherapy in FLT3-ITD-mutated, relapsed or refractory AML. Blood 132, 598-607 (2018) 
36. Cortes, J. et al. Quizartinib, an FLT3 inhibitor, as monotherapy in patients with relapsed or refractory acute myeloid leukaemia: an open-label, multicentre, single-arm, phase 2 trial. Lancet Oncol. 19, 889-903 (2018).

37. Cortes, J. E. et al. Phase I study of quizartinib administered daily to patients with relapsed or refractory acute myeloid leukemia irrespective of FMS-like tyrosine kinase 3-internal tandem duplication status. J. Clinl. Oncol. 31, 3681 (2013).

38. Perl, A. E. et al. Final results of the chrysalis trial: a first-in-human phase $1 / 2$ dose-escalation, dose-expansion study of gilteritinib (ASP2215) in patients with relapsed/refractory acute myeloid leukemia (R/R AML). Blood 128, 1069 (2016).

39. Perl, A. E. et al. Selective inhibition of FLT3 by gilteritinib in relapsed or refractory acute myeloid leukaemia: a multicentre, first-in-human, open-label, phase 1-2 study. Lancet Oncol. 18, 1061-1075 (2017).

40. Perl, A. E. et al. Gilteritinib or chemotherapy for relapsed or refractory FLT3mutated AML. N. Engl. J. Med. 381, 1728-1740 (2019).

41. Metzelder, S. et al. High activity of sorafenib in FLT3-ITD-positive acute myeloid leukemia synergizes with allo-immune effects to induce sustained responses. Leukemia 26, 2353-2359 (2012).

42. Burchert, A. et al. Sorafenib maintenance after allogeneic hematopoietic stem cell transplantation for acute myeloid leukemia with FLT3-internal tandem duplication mutation (SORMAIN). J. Clin. Oncol. 38, 2993-3002 (2020).

43. Xuan, L. et al. Sorafenib maintenance in patients with FLT3-ITD acute myeloid leukaemia undergoing allogeneic haematopoietic stem-cell transplantation: an open-label, multicentre, randomised phase 3 trial. Lancet Oncol. 21, 1201-1212 (2020).

44. Pratz, K. W. et al. A phase 1 study of gilteritinib in combination with induction and consolidation chemotherapy in patients with newly diagnosed AML: final results. Blood 136, 16-17 (2020).

45. Wang, E. S. et al. Phase 3, Multicenter, Open-label Study of Gilteritinib, Gilteritinib plus Azacitidine, or Azacitidine Alone in Newly Diagnosed FLT3-Mutated (FLT3 mut ${ }^{+}$) Acute Myeloid Leukemia (AML) Patients Ineligible for Intensive Induction Chemotherapy (ASH, 2020).

46. Astellas Reports XOSPATA ${ }^{\circledR}$ (gilteritinib) in combination with azacitidine did not meet endpoint of overall survival in newly diagnosed flt3 mutationpositive acute myeloid leukemia patients ineligible for intensive induction chemotherapy (2020).

47. Strati, P. et al. Phase I/II trial of the combination of midostaurin (PKC412) and 5azacytidine for patients with acute myeloid leukemia and myelodysplastic syndrome. Am. J. Hematol. 90, 276-281 (2015).

48. Swaminathan, M. et al. The combination of quizartinib with azacitidine or low dose cytarabine is highly active in patients (Pts) with FLT3-ITD mutated myeloid leukemias: interim report of a phase I/II trial. Blood 130, 723 (2017).

49. Mali, R. S. et al. Venetoclax combines synergistically with FLT3 inhibition to effectively target leukemic cells in FLT3-ITD+ acute myeloid leukemia models. Haematologica 106, 1034 (2020).

50. Brinton, L. T. et al. Synergistic effect of BCL2 and FLT3 co-inhibition in acute myeloid leukemia. J. Hematol. Oncol. 13, 139 (2020).

51. Zhu, R., Li, L., Nguyen, B., Duffield, A. S. \& Small, D. Gilteritinib and venetoclax synergize to eliminate FLT3/ITD+ leukemia cells through BIM. Blood 134, 2564 (2019).

52. Yamatani, $\mathrm{K}$. et al. Upregulation of Bcl-2 confers resistance to FLT3 inhibition in FLT3-ITD AML with secondary acquired mutations. Blood 132, 3944 (2018).

53. Naval Daver, M. D. et al. Efficacy and Safety of Venetoclax in Combination with Gilteritinib for Relapsed/refractory FLT3-mutated Acute Myeloid Leukemia in the Expansion Cohort of a Phase 16 Study (ASH, 2020).

54. DiNardo, C. D. et al. 10-day decitabine with venetoclax for newly diagnosed intensive chemotherapy ineligible, and relapsed or refractory acute myeloid leukaemia: a single-centre, phase 2 trial. Lancet Haematol. 7, e724-e736 (2020).
55. Abhishek Maiti, M. D. et al. Venetoclax, FLT3 Inhibitor and Decitabine in FLT3mut Acute Myeloid Leukemia: Subgroup Analysis of a Phase II Trial (ASH, 2020).

56. Musa Yilmaz, M. et al. Quizartinib with Decitabine +/-Venetoclax is Highly Active in Patients (Pts) with FLT3-ITD Mutated (mut) Acute Myeloid Leukemia (AML): Clinical Report and Signaling Cytof Profiling from a Phase IB/II Trial (ASH, 2020).

57. Burnett, A. K. Russell, N. H. \& Hills, R. K. Group obotUKNCRIAMLS. Higher daunorubicin exposure benefits FLT3 mutated acute myeloid leukemia. Blood 128, 449-452 (2016)

58. Libura, M. et al. Cladribine added to daunorubicin-cytarabine induction prolongs survival of FLT3-ITD+ normal karyotype AML patients. Blood 127, 360-362 (2016).

59. Altman, J. K. et al. Phase 1 study of quizartinib in combination with induction and consolidation chemotherapy in patients with newly diagnosed acute myeloid leukemia. Am. J. Hematol. 93, 213-221 (2018).

60. Wang, E. S. et al. Low relapse rate in younger patients $\leq 60$ years old with newly diagnosed FLT3-mutated acute myeloid leukemia (AML) treated with crenolanib and cytarabine/anthracycline chemotherapy. Blood 130, 566 (2017).

61. Jain, $P$. et al. Cladribine combined with idarubicin and Ara-C (CLIA) as a frontline and salvage treatment for young patients ( $\leq 65 \mathrm{yrs}$ ) with acute myeloid leukemia. Blood 128, 1639 (2016).

62. Sasaki, K. et al. Sorafenib plus intensive chemotherapy improves survival in patients with newly diagnosed, FLT3-internal tandem duplication mutationpositive acute myeloid leukemia. Cancer 125, 3755-3766 (2019).

63. Kadia, T. et al. Venetoclax Added to Cladribine, Idarubicin, and Cytarabine with or without a FLT3 Inhibitor in Newly Diagnosed Acute Myeloid Leukemia (EHA, 2020).

64. DiNardo, C. D. et al. Azacitidine and venetoclax in previously untreated acute myeloid leukemia. N. Engl. J. Med. 383, 617-629 (2020)

65. Konopleva, M. et al. Results of venetoclax and azacitidine combination in chemotherapy ineligible untreated patients with acute myeloid leukemia with FLT3 mutations. Blood 136, 8-10 (2020).

66. Chyla, B. et al. Genetic biomarkers of sensitivity and resistance to venetoclax monotherapy in patients with relapsed acute myeloid leukemia. Am. J. Hematol. 93, E202-E205 (2018).

67. DiNardo, C. D. et al. Molecular patterns of response and treatment failure after frontline venetoclax combinations in older patients with AML. Blood 135 791-803 (2020)

68. Daver, N. et al. Secondary mutations as mediators of resistance to targeted therapy in leukemia. Blood 125, 3236-3245 (2015).

69. Smith, C. C. et al. Validation of ITD mutations in FLT3 as a therapeutic target in human acute myeloid leukaemia. Nature 485, 260-263 (2012).

70. Alotaibi, A. S. et al. Patterns of resistance differ in patients with acute myeloid leukemia treated with type I versus type II FLT3-inhibitors. Blood Cancer Discov. 2, 125 (2020)

71. McMahon, C. M. et al. Clonal selection with RAS pathway activation mediates secondary clinical resistance to selective FLT3 inhibition in acute myeloid leukemia. Cancer Discov. 9, 1050-1063 (2019).

72. Alotaibi, A. S. et al. Emergence of BCR-ABL1 fusion in AML post-FLT3 inhibitorbased therapy: a potentially targetable mechanism of resistance-a case series. Front. Oncol. 10, 588876- (2020).

73. Yilmaz, M. et al. Outcomes with sequential FLT3-inhibitor-based therapies in patients with AML. J. Hematol. Oncol. 13, 132 (2020).

74. Perl, A. E. et al. Clinical outcomes in patients with relapsed/refractory acute myeloid leukemia treated with gilteritinib who received prior midostaurin or sorafenib. Blood 136, 22-23 (2020). 